\title{
O consumo de naguilé em acadêmicos de medicina
}

\author{
The consumption of hookah by medicine academics \\ El consumo de naguilé en medicina académica
}

\begin{abstract}
RESUMO
Objetivo: Caracterizar a prevalência e o uso do narguilé entre acadêmicos do curso de medicina. Método: Trata-se de um estudo transversal, descritivo, quantitativo realizado com acadêmicos do curso de medicina de uma instituição de ensino superior privada do Sul do país. Nesse contexto, foi aplicado um questionário estruturado sobre perfil sociodemográfico e uso do narguilé. Resultados: Participaram da pesquisa 247 acadêmicos; 53,4\% do sexo feminino. Destes, 98 (39,8\%) referiram ter feito ou fazer uso do narguilé, sendo que 39,8\% iniciaram o uso com idades entre 17 e 19 anos; e $57,2 \%$ o usam por diversão; $99 \%$ não se consideram dependentes; $23,5 \%$ utilizam substâncias ilícitas concomitante; $22,4 \%$ tem acesso no próprio meio familiar, $35,7 \%$ custeiam o uso pela renda dos familiares e $12,1 \%$ consentem sobre o uso; tosse e hipotensão foram os sinais referidos durante o consumo (36,5\% e 14,7\%, respectivamente). Conclusão: Conclui-se que a prevalência do uso de narguilé foi considerável e enfatizam a necessidade de uma abordagem nas instituições de ensino. DESCRITORES: Tabagismo; Uso de Tabaco; Consumo de Produtos Derivados do Tabaco; Tabaco para Cachimbos de Água; Prevalência.
\end{abstract}

\section{ABSTRACT}

Objective: To characterize the prevalence and use of hookah among medical students. Method: This is a cross-sectional, descriptive, quantitative study carried out with medical students from a private higher education institution in the south of the country. In this context, a structured questionnaire was applied on sociodemographic profile and use of hookah. Results: 247 academics participated in the research; $53.4 \%$ were female. Of these, $98(39.8 \%)$ reported having used or using the hookah, $39.8 \%$ of whom started using it between the ages of 17 and 19; and 57.2\% use it for fun; $99 \%$ do not consider themselves dependent; $23.5 \%$ use illicit substances concomitantly; $22.4 \%$ have access in the family environment, $35.7 \%$ finance the use for the family's income and $12.1 \%$ consent to the use; cough and hypotension were the signs reported during consumption (36.5\% and $14.7 \%$, respectively). Conclusion: We conclude that the prevalence of hookah use was considerable and emphasize the need for an approach in educational institutions.

ESCRIPTORS: Smoking; Tobacco Use; Consumption of Tobacco Derived Products; Tobacco for Hookahs; Prevalence.

\section{RESUMEN}

Objetivo: Caracterizar la prevalencia y el uso de narguile entre estudiantes de medicina. Método: Se trata de un estudio transversal, descriptivo y cuantitativo realizado con estudiantes de medicina de una institución privada de educación superior en el sur del país. En este contexto, se aplicó un cuestionario estructurado sobre perfil sociodemográfico y uso de narguile. Resultados: 247 académicos participaron en la investigación; El 53,4\% eran mujeres. De estos, 98 (39,8\%) informaron haber usado o usar la pipa de agua, de los cuales el 39,8\% comenzó a usarla entre los 17 y 19 años; y el 57,2\% lo usa para divertirse; El 99\% no se considera dependiente; El 23,5\% consume sustancias ilícitas de forma concomitante; El 22,4\% tiene acceso en el ámbito familiar, el $35,7 \%$ financia el uso para el ingreso familiar y el 12,1\% consiente el uso; tos e hipotensión fueron los signos reportados durante el consumo (36,5\% y 14,7\%, respectivamente). Conclusión: Concluimos que la prevalencia del uso de narguile fue considerable y enfatizamos la necesidad de un enfoque en las instituciones educativas.

DESCRIPTORES: Fumar; El consumo de tabaco; Consumo de productos derivados del tabaco; Tabaco para narguiles; Predominio.

RECEBIDO EM: 15/09/2020 APROVADO EM: 06/10/2020

\section{Renata de Oliveira Nodari}

Acadêmica do curso de Medicina, Centro Universitário de Maringá -UNICESUMAR.

ORCID: 0000-0001-6711-5521 


\section{Maria Livien Kubaski}

Acadêmica do curso de Medicina, Centro Universitário de Maringá - UNICESUMAR. ORCID: 0000-0002-7173-0698

\section{Eduardo Rocha Covre}

Doutorando em Enfermagem, Docente do Programa de Pós Graduação em Enfermagem pela Universidade Estadual de Maringá. ORCID: 0000-0001-7338-121X

\section{Maria Aparecida Salci}

Doutora em Enfermagem, Docente do Programa de Pós Graduação em Enfermagem pela Universidade Estadual de Maringá. ORCID: 0000-0002-6386-1962

\section{Ludmila Lopes Maciel Bolsoni}

Doutora em Enfermagem, Docente do Programa de Pós Graduação em Enfermagem pela Universidade Estadual de Maringá. Docente do curso de Medicina, Centro Universitário de Maringá - UNICESUMAR.

ORCID: 0000-0002-1645-8572

\section{Patricia Bossolani Charlo}

Doutora em Enfermagem, Docente do Programa de Pós Graduação em Enfermagem pela Universidade Estadual de Maringá. Docente do curso de Medicina/Enfermagem, Centro Universitário de Maringá - UNICESUMAR.

ORCID: 0000-0002-8262-2086

\section{INTRODUÇÃO}

0 tabagismo é uma doença crônica caracterizada pela dependência à nicotina presente nos produtos à base de tabaco, e continua a ser um importante problema de saúde pública considerado como a principal causa evitável isolada de adoecimento e mortes precoces no mundo. ${ }^{(1-3)}$ Segundo a Organização Mundial da Saúde (OMS), o tabaco mata mais de 8 milhões de pessoas por ano e a maioria das mortes é resultado do consumo direto do produto. ${ }^{(4)}$ Evidências apontam que o consumo do tabaco tem sido consideravelmente disseminado por diversos países no mundo e que a maioria dos indivíduos iniciam o seu consumo já na adolescência ou até mesmo antes dos 20 anos de idade. $\mathrm{O}$ consumo precoce do tabaco está relacionado a diversos fatores, com destaque para como às condições sociodemográficas dos indivíduos (idade, sexo e religião); às relações familiares conflitantes, como também a influência de pais e irmãos fumantes, ausência de apoio, participação e acompanhamento da família nas atividades; às relações sociais como influência dos amigos; e aos fatores comportamentais como o uso de drogas ilícitas e início da atividade sexual. ${ }^{5}$ Existem diversas formas de consumir o tabaco, porém fumá-lo é a prática mais comum e pode ser realizada por meio do uso de cachimbos, charutos, cigarros e/ou narguilé que também é chamado de cachimbo d'água. ${ }^{6}$ O narguilé foi inventado na Índia por um médico chamado Hakim Abul Fath, que sugeriu passar o fumo antes de ser inalado em um pequeno recipiente com água, como um processo mais seguro e menos nocivos à saúde humana. Essa suposição estimulou a propagação do conhecimento popular, de que o narguilé seria mais vantajoso que os outros produtos do tabaco. Entretanto, evidências científicas disponíveis na atualidade não sustentam mais essa hipótese. Assim, a crença popular ilegítima, aliada às propagandas que incentivam o uso do narguilé, somado ao acesso facilitado ao equipamento e seus produtos, aos inúmeros artifícios atrativos ao uso como a inclusão de essências com sabores e aromas ao fumo, e às medidas de socialização implícita no consumo são atrativos que justificam o uso indiscriminado, principalmente entre os jovens. ${ }^{8}$ Com isso, o número de adeptos ao uso do equipamento vem crescendo substancialmente e tem se tornado epidêmico entre os adolescentes, gerando um alerta para a Organização Mundial de Saúde (OMS) e sociedade médica. ${ }^{9}$ Atualmente, há evidencias que nas Universidades, os acadêmicos buscam o bem-estar e a socialização por meio do uso de substâncias e equipamentos como o narguilé, visto que neste período eles são expostos a diversos fatores estressantes. ${ }^{10}$ Para responder a questão de pesquisa: Qual é a prevalência e a característica do uso do narguilé entre os acadêmicos do curso de medicina? E, para preencher uma lacuna do conhecimento a fim de produzir informações que auxiliem na elaboração de estratégias de prevenção e educação em saúde voltadas para usuários do narguilé conduziu-se o presente estudo, que teve como objetivo caracterizar a prevalência e o uso do narguilé entre acadêmicos do curso de medicina.

\section{MÉTODO}

Trata-se de um estudo transversal, descritivo, de abordagem quantitativa realizado com acadêmicos do curso de medicina de uma Instituição de Ensino Superior (IES) privada localizada em um município de médio porte na região Sul do país, durante o período de fevereiro a abril de 2019. No período da coleta de dados estavam matriculados da primeira a sexta série do curso de medicina da IES 988 alunos (380 no $1^{\circ}$ ano; 220 no $2^{\circ}$ ano; 100 no $3^{\circ}$ ano; 96 no $4^{\circ}$ ano; 96 no $5^{\circ}$ ano e 96 no $6^{\circ}$ ano). Para selecionar os sujeitos da pesquisa foi realizado o processo de amostragem probabilística do tipo estratificada uni- 
forme. Para a composição da amostra foi utilizado como critério de seleção $25 \%$ do total de acadêmicos de cada série do curso (a saber: 95 alunos do $1^{\circ}$ ano, 55 alunos do $2^{\circ}$ ano, 25 alunos do $3^{\circ}$ ano, 24 alunos do $4^{\circ}$ ano, 24 alunos do $5^{\circ}$ ano e 24 alunos do $6^{\circ}$ ano). Em seguida, por meio de amostra- gem aleatória simples os participantes de cada série foram recrutados. Para compor a amostra foram incluídos os acadêmicos devidamente matriculados nas respectivas séries e maiores de 18 anos de idade. Não houve perdas amostrais, totalizando uma amostra de $n=247$ acadêmicos. Para a co-

Tabela 1. Caracterização do uso do narguilé entre acadêmicos do curso de medicina de uma Universidade privada. Maringá/PR, 2019.

\section{Características \\ Fazem uso do narguilé}

Sim

Não

Idade de início do uso

11-13 anos

$14-16$ anos

17-19 anos

20-22 anos

23-25 anos

Frequência do uso

Todos os dias

Raramente

Somente nos fins de semana

Somente em festas

Outro

Motivo do uso

Alívio

Prazer

Diversão

Pressão social

Mais de uma opção

Origem do equipamento para o uso

Próprio

Tabacarias

Outra procedência

\section{Renda para custear o uso}

Trabalho

Renda familiar

Amigos

Outros

Mais de uma opção

Fonte: próprio autor, 2019.
N

$n(\%)$

247

$98(39,8)$

$149(60,2)$

98

$04(4,1)$

$29(29,6)$

$39(39,8)$

$20(20,4)$

$06(6,1)$

98

$02(2,0)$

$59(60,2)$

$16(16,4)$

$19(19,4)$

$02(2,0)$

98

$01(1,0)$

$28(28,6)$

$56(57,2)$

$01(1,0)$

$12(12,2)$

98

$22(22,4)$

$13(13,3)$

$63(64,3)$

98

$02(2,0)$

$35(35,7)$

$29(29,6)$

$27(27,6)$

$05(5,1)$ leta de dados foi utilizado um questionário estruturado com 16 questões objetivas e de múltipla escolha sobre o perfil sociodemográfico e do uso do narguilé entre os acadêmicos do curso de medicina. Posteriormente, os dados foram analisados estatisticamente no Microsoft Office Excel 2016. A análise descritiva foi apresentada por meio de tabelas com frequência absoluta (n) e frequência relativa (\%). O estudo respeitou os aspectos éticos das resoluções 466/2012 e 510/2016 do Conselho Nacional de Saúde, 11 com aprovação do Comitê de Ética e Pesquisa sob parecer número 3.076.436 e conforme exposto na Plataforma Brasil número do CAAE 03711318.8.0000.5539.A participação dos sujeitos foi validada após o aceite, leitura e assinatura do Termo de Consentimento Livre e Esclarecido (TCLE).

\section{RESULTADOS}

Participaram do estudo 247 acadêmicos de medicina, destes $53,4 \%$ do sexo feminino e $46,6 \%$ masculinos; com idade entre 18 e 20 anos (49,8\%), 21 e 22 anos $(23,1 \%)$ e, 23 anos ou mais $(27,1 \%)$. Do total de acadêmicos que compuseram a amostra 98 (39,8\%) referiram ter feito ou fazer uso do narguilé. Deste, 39,8\% iniciaram o uso com idades entre 17 e 19 anos e 29,6\% com idades entre 14 e 16 anos. Porém, quando questionados em relação a frequência com que os mesmos utilizam o equipamento, $60,2 \%$ afirmaram ser raramente e $57,2 \%$ justificaram o uso a um momento de diversão/lazer. Ainda, 99\% desses acadêmicos não se consideraram dependentes em relação ao uso do narguilé e 23,5\% referiram utilizar substâncias ilícitas concomitante a esse consumo. Em relação ao acesso dos acadêmicos aos materiais e equipamentos próprios para o uso do narguilé, 22,4\% afirmaram que possuíam em casa os artefatos, $13,3 \%$ frequentavam tabacarias e $64,3 \%$ afirmaram consegui-lo de outras formas, como por exemplo empréstimo do equipamento por meio dos amigos. O custeio do uso do narguilé de $35,7 \%$ dos acadêmicos ocorria com o apoio da renda familiar (tabela 1). 


\section{artigo}

Nodari, R.O.; Kubaski, M.L.; Covre, E.R.; Salci, M.A.; Bolsoni, L.L.M.; Charlo, P.B.;

O consumo de naguilé em acadêmicos de medicina

A respeito dos possíveis sinais e sintomas que os usuários do narguilé poderiam apresentar durante o seu uso, $36,5 \%$ dos acadêmicos referiram hipotensão, $14,7 \%$ tosse e $24,5 \%$ nenhum dos sintomas sugeridos. Cabe destacar que nessa questão o aluno poderia assinalar mais de uma opção se julgasse pertinente (tabela 2).Por fim, observa-se que quando questionados a respeito da aceitação dos familiares em relação ao uso do narguilé $40,9 \%$ dos acadêmicos não souberam responder, enquanto que $25,9 \%$ referiram que os familiares não aceitavam o uso do narguilé, $13 \%$ afirmaram que não conversavam sobre o assunto e $12,1 \%$ conheciam e aceitavam o uso (tabela 2).

Tabela 2. Associação do uso do narguilé e os principais sintomas respectivos ao consumo do narguilé. Maringá/PR, 2019.

Características

Dependência do uso

Sim

Não

Uso de substância ilícita concomitante

Sim

Não

Sinais e sintomas apresentados durante o uso*

Hipotensão

Taquicardia

Pigarro

Cefaléia

Lesões na mucosa bucal

Dispnéia

Tosse

Nenhum dos sintomas

Aceitação familiar sobre o uso

Aceitam

Não aceitam

Falam abertamente sobre o uso

Nunca se referiram ao assunto

Não responderam

Fonte: próprio autor, 2019.

*O acadêmico poderia assinalar mais de uma opção.

\section{DISCUSSÃO}

De acordo com a prevalência do uso de narguilé entre os participantes desse estudo, aproximadamente $40 \%$ dos acadêmicos faziam ou já fizeram o uso do equipamento em algum momento de suas vidas. Estudo similar a esse conduzido em 2013 nos Estados Unidos traz dados semelhantes sobre a prevalência do uso do narguilé entre estudantes, na qual estimou uma frequência de experimentação de $46,4 \%$ e de uso contínuo de $28,4 \%$ no período de um ano entre os 1.023 participantes. $12 \mathrm{No}$ Brasil, um estudo realizado em uma Universidade privada de Curitiba/PR que investigou a prevalência do uso de produtos derivados do tabaco entre universitários do curso de odontologia, evidenciou uma prevalência de aproximadamente $25 \%$ de uso do tabaco entre os estudantes, sendo que o narguilé mostrou-se como o produto de maior consumo em quase $67 \%$ dos participantes. ${ }^{13}$

Outro estudo conduzido em 2018, que utilizou dados da Pesquisa Nacional de Saúde do Escolar (PeNSE) de 2012 e 2015 e objetivou identificar a frequência e os fatores associados ao uso do narguilé e outros produtos derivados do tabaco entre os escolares brasileiros, mostrou que no ano de 2015 o narguilé foi o produto mais utilizado entre os alunos $(71,6 \%)$. Além disso, mostrou ainda que estudar em instituição privada de ensino foi fator positivo para o consumo do tabaco e outros derivados. ${ }^{14} \mathrm{~A}$ alta prevalência do uso do narguilé, principalmente entre os jovens, pode ser justificado pela forte disseminação do uso do equipamento no Brasil nas últimas décadas e pelas propagandas atrativas incentivando o seu consumo, o acesso facilitado ao equipamento e ao fato de ser considerado um meio de socialização entre os jovens8. Diante desse contexto, associando ao aumento do uso do narguilé entre os brasileiros, em 2015, no Dia Nacional do Combate ao Fumo, a campanha teve como tema o Narguilé e a Iniciação ao Fumo, com alertar sobre os perigos de fumar. Segundo o Ministério da Saúde em um período de cinco anos analisados, o percentual de adesão ao cachimbo d'água mais que dobrou, principalmente em indivíduos do sexo masculino. ${ }^{15} \mathrm{~A}$ respeito do início do uso do narguilé entre os participantes desse estudo, os acadêmicos relataram ter iniciado, em sua maioria, com idades entre 14 e 19 anos de idade (70\% da somatória total, aproximadamente), ou seja, essa faixa etária de idade coincidi com o período de transição entre o Ensino Médio e o ingresso no Ensino Superior. Esses achados corroboram com evidências trazidas pela literatura, mostrando que a maioria dos indivíduos iniciam o consumo do tabaco na adolescência ou até mesmo antes dos 20 anos de idade.13Ainda, quando questionados a respeito da frequência do uso do narguilé, mais de $60 \%$ afirmaram usá-lo de forma esporádica (raramente). 
Estudo realizado em 2019, com acadêmicos do Centro Universitário de Brasília (UniCEUB), sobre a prevalência e o perfil dos estudantes que utilizavam narguilé, identificou que mais de $60 \%$ dos participantes já haviam experimentado o produto e, aproximadamente $36 \%$ raramente faziam o uso do equipamento por cerca de 20 minutos. ${ }^{16}$ A frequência com que os jovens utilizam o narguilé para o consumo de produtos derivados do tabaco são preocupantes, pois mesmo que a utilização do equipamento seja rara, as evidências apontam que uma tragada no equipamento é semelhante ao volume de fumaça inalada com um único cigarro, ou seja, o narguilé é mais prejudicial à saúde e em uma única sessão pode ser equivalente a fumar de 20 a 30 cigarros. ${ }^{17}$ Nesse estudo, quando os participantes foram questionados sobre os motivos que os levam ao uso do narguilé, $57 \%$ justificaram ou associaram a um momento de lazer e diversão. Levando em consideração esse argumento, evidências trazem que as principais motivações que atraem os jovens ao narguilé se devem ao fato do mesmo oferecer uma variedade de sabores e aromas e não ser considerado, por muitos usuários, como uma forma de fumar tabaco. ${ }^{16}$ Isso faz com que a prática do uso do narguilé favoreça a socialização, o convívio com amigos e os momentos considerados de descontração, como foi apontado em outros estudos. ${ }^{18}$ Em relação ao acesso dos acadêmicos aos materiais e equipamentos próprios para o uso do narguilé, observou-se que mais de $35 \%$ o têm de forma facilitada por meio de tabacarias ou pelo próprio ambiente familiar. Contudo, mais de $64 \%$ consegue o narguilé por outros meios não mencionados. Um estudo realizado com acadêmicos de odontologia refere que os alunos conseguiam comprar ou consumir o narguilé de forma facilitada em postos de combustíveis, bares e bancas de jornal. ${ }^{13}$ Diante disso, autoridades de saúde e de segurança dos municípios devem intervir nos ambientes comerciais supramencionados, por meio de ações de educação em saúde voltadas para os usuários de narguilé quanto aos seus malefícios para a saúde humana. Além disso, ações de fiscalização seriam importantes para monitorar o consumo de substâncias ilícitas junto ao uso do narguilé.

No Brasil durante o período 2003 a 2015, um estudo utilizou o uso de inquéritos populacionais apoiando políticas públicas baseadas em evidências. Os estudos trouxeram resultados da Pesquisa Nacional de saúde na escola e da Pesquisa Nacional de Saúde (PNS) de 2013, que orientaram a regulamentação da Lei Antitabagismo, como o decreto presidencial sobre ambientes livres de tabaco, o aumento da taxação e o estabelecimento de preço mínimo do tabaco, a portaria interministerial que incluiu o narguilé na regulamentação e proibição em ambientes livre do fumo, em 2014. ${ }^{21}$ Cabe ressaltar que a implantação e manutenção destas medidas é fundamental para os avanços na prevenção de agravos a saúde, permitindo estratégias como a fiscalização dos ambientes livres de tabaco, atenção para as tendências e os modismos de novos produtos .Essas estratégias visam impedir ou retardar a iniciação de tabaco entre jovens, como um dos objetivos da saúde pública. ${ }^{21}$ Ainda sobre a acessibilidade ao equipamento, $35,7 \%$ dos acadêmicos referiram que o custeio para o uso do narguilé e seus produtos se dá por meio da renda familiar dos participantes, ou seja, em parte os familiares tem conhecimento do uso pelo responsável. Adicionalmente, quando questionados sobre a opinião dos familiares em relação ao uso do equipamento, $25,9 \%$ referiram que os mesmos não aceitavam, $13 \%$ afirmaram que não dialogavam sobre o assunto e $12,1 \%$ aceitavam o uso sem impedimentos. Diante disso, fica evidente a necessidade de se discutir sobre essa temática no meio familiar e orientá-los quanto aos futuros danos que o uso do narguilé pode trazer para a saúde, pois é sabido que o consumo do tabaco via narguilé também está associado a um maior risco do desenvolvimento de doenças crônicas, principalmente as pulmonares, periodontal, câncer de pulmão e depen- dência da nicotina.8,23 A nicotina presente nos produtos à base de tabaco causa dependência física e psicológica em seus usuários ${ }^{17}$. Quando os participantes desse estudo foram questionados quanto a dependência ou não do uso do narguilé, $99 \%$ dos que já haviam utilizado não se consideravam dependentes do equipamento. Contudo, estudo similar que objetivou verificar a prevalência de uso/ experimentação de narguilé e fatores associados em estudantes de uma universidade também do Sul do Brasil mostrou que indivíduos que fumaram narguilé pela primeira vez antes dos 18 anos e que utilizavam tabaco saborizado, apresentaram mais chances de permaneceram em sessões mais longas de narguilé. ${ }^{22}$

Esses dados revelam, de forma subliminar, que o consumo do narguilé também leva à dependência tabágica, mesmo que os usuários não a reconheça. Além do significativo aumento do consumo do narguilé, outro fator preocupante é a associação de substâncias ilícitas e consumo de álcool, concomitantemente ao uso desse. O consumo dessas substâncias vem aumentando entre os jovens universitários, que eventualmente se encontram em uma fase de conflitos cognitivos e afetivos e se deparam com um ambiente propício para isso. Esse estudo mostrou que quase 24\% dos acadêmicos que utilizavam o narguilé afirmaram utilizar outras substâncias ilícitas concomitantemente. Trata-se de um dado que gera preocupação, tendo em vista que as drogas alteram o nível de consciência, levando os indivíduos a se envolverem em situações de sexo sem preservativo, violência, além de causar problemas no meio familiar e dependência química. ${ }^{16}$ Nesse estudo, os usuários do narguilé referiram sintomatologias que acompanham o consumo do produto e as que o sucedem, evidenciando que mesmo provoca toxicidade ao organismo. Sintomas como hipotensão $(36,5 \%)$ e tosse $(14,7 \%)$ foram os mais frequentes. Estudo realizado em 2017 que identificou a prevalência e o perfil de estudantes que utilizavam narguilé das Faculdades Integradas Padre Albino (FIPA), mostrou que os participantes 


\section{artigo}

Nodari, R.O.; Kubaski, M.L.; Covre, E.R.; Salci, M.A.; Bolsoni, L.L.M.; Charlo, P.B.;

O consumo de naguilé em acadêmicos de medicina.

também referiram tontura, cefaleia, fraqueza, náuseas, dispneia e vômitos durante e após o consumo de narguilé. Durante o uso do narguilé foi constatado que, além de não ocorrer a queima do carvão, ocorre a combustão incompleta do tabaco, visto que essa acontece em uma temperatura próxima a $500^{\circ} \mathrm{C}$ (inferior à temperatura de combustão do cigarro comum), essa temperatura elevada favorece a liberação de concentrações de produtos tóxicos, as quais são inaladas pelos usuários (tanto pelos fumantes ativos quanto pelos passivos). Cerca de 300 produtos químicos fo- ram identificados na fumaça do narguilé, e quantificadas 82 substâncias tóxicas, 23 cancerígenas, além da nicotina que causa dependência. ${ }^{15}$

\section{CONCLUSÃO}

Esse estudo trouxe contribuições significativas sobre a prevalência e característica do uso do narguilé entre os acadêmicos do curso de medicina. Entende-se que essa temática é de grande importância para a saúde pública, tendo em vista a crescente prevalência do uso do narguilé entre os jovens e também as inúmeras evidências científicas que abordam sobre os malefícios que o mesmo traz para a saúde física, psicológica e familiar. Diante disso, é de suma importância abordar sobre o assunto nas instituições de ensino, de todos os níveis, e conscientizar os alunos quanto aos danos que o uso do narguilé provoca no organismo a curto, médio e longo prazo. Os resultados obtidos neste estudo têm grande relevância social, contudo, como limitações pontua-se que o estudo transversal inviabiliza delinear relações de causalidade.

\section{REFERÊNCIAS}

1. World Health Organization [homepage on the Internet]. WHO, Geneva; Fact Sheet [updated 2018 Mar 9, cited 2018 Aug 26].

2. Drope J, et al. The Tobacco Atlas. Atlanta: American Cancer Society and Vital Strategies.2018.

3. Ministério da Saúde/INCA. Tabagismo. Disponível: https:// www.inca.gov.br/tabagismo

4. World Health Organization. Tobacco. Available at: https://www. who.int/news-room/fact-sheets/detail/tobacco.

5. Silva,V. L., et. al. Determinantes do consumo de tabaco por estudantes. Rev. Saúde Pública. 2017 ;51:36. Epub May 04, 2017.

6. OPAS. Organização Pan-Americana de Saúde. 2019. Disponível:https://www.paho.org/bra/index.php?option=com_ content \&view=article \&id=5641: folha-informativa-tabaco\&ltemid=1097

7. Brasil. Ministério da Saúde. Instituto Nacional de Câncer José Alencar Gomes da Silva. Rio de Janeiro: INCA; 2019. Narguilé: o que sabemos?

8. Ministério da Saúde. Instituto Nacional de Câncer José Alencar Gomes da Silva (INCA). Uso de narguilé: efeitos sobre a saúde, necessidades de pesquisa e ações recomendadas para legisladores. Grupo de Estudo da OMS sobre a Regulação de Produtos de Tabaco (TobReg). 2a ed. Rio de Janeiro, 2017.

9. Shihadeh, A., et.al. Toxicant content, physical properties and biological activity of waterpipe tobacco smoke and its tobacco-free alternatives. Tob Control,2015.

10. Silva M. IG, et al. Uso de substâncias psicoativas entre estudantes da área da saúde. In: $6^{\circ}$ Congresso Internacional em Saúde. 2019.

11. Brasil. Conselho Nacional de Saúde. Resolução n 466, de 12 de dezembro de 2012. Regulamenta as diretrizes e normas regulamentadoras de pesquisas envolvendo seres humanos. Diário Oficial da União. Brasília, DF, 12 dez. 2012.

12. Barnett T.E., et al. Evidence of emerging hookah use among university students: a crosssectional comparison between hoo- kah and cigarette use. BMC Public Health. 2013; 13(1):302.

13. Beckert N, et al. Características do uso de produtos derivados do tabaco entre universitários do curso de odontologia em uma universidade de Curitiba. Rev. Odontol. UNESP. 2016, 45(1): 7-14. https://doi.org/10.1590/1807-2577.10015

14. Malta DC, et al. Factors associated with the use of waterpipe and other tobacco products among students, Brazil, 2015. Rev Bras Epidemiol 2018, 21(1): E180006.

15. Brasil.Ministério da Saúde. Mais de 212 mil brasileiros admitem usar narguilé. Set, 2015.

16. Tavares, I.B, et.al. Prevalência e perfil de universitários que fumam narguilé no Distrito Federal. Programa de Iniciação Científica, 2018.

17. Eriksen M., et.al. The Tobacco Atlas,5th ed. Atlanta: American Cancer Society; 2015.

18. Abdulrashid, O. A., et.al. Factors contributing to the upsurge of water-pipe tobacco smoking among Saudi femalesin selected Jeddah cafés and restaurants: A mixed method study. JFCM, 2018, 25(1):13-19.

19. Reis, A.A.C.D., et. al. Desafios para as políticas públicas voltadas à adolescência e juventude a partir da Pesquisa Nacional de Saúde do Escolar (PeNSE). Cien Saúde Colet. 2018;23(9):2879 2890.

20. Akl E.A., et. al. The effects of waterpipe tobacco smoking on health outcomes: a systematic review. Int J Epidem. 2010; 39(3):834-57.

21. Santos K., et.al. Prevalência de uso de narguilé e fatores associados em universitários.2019.

22. Magri M.A, et al. Estudo do uso de narguilé entre estudantes de Medicina de uma faculdade do noroeste paulista. Ciência, Pesquisa e Consciência 2017, 9(1):25-30.

23. Torrey CM, et al. Waterpipe cafes in Baltimore, Maryland: Carbon monoxide, particulate matter, and nicotine exposure. JESEE. 2015; 25(4):405-10. 